\title{
Design and Construction of an Arduino Microcontroller- based EGG Incubator
}

\author{
Frimpong Kyeremeh \\ Sunyani Technical University, Ghana
}

\author{
Forson Peprah \\ Volta River Authority, Brong-Ahafo, Ghana
}

\begin{abstract}
Meeting the high demand for poultry products calls for the use of artificial egg hatcheries but the backyard and small-scale poultry farmers are constrained by the dependence on natural incubation or on commercial hatcheries for young birds for breeding. In this paper an incandescent bulb heat source incubator is designed and constructed to hatch 14000 quail eggs (4500 chicken egg equivalent). The incubator system is an Arduino microcontroller-based, which controls the heaters, air circulation fans and the mechanism for turning the trays, through relays. The prevailing conditions in the incubator (temperature and the humidity) are displayed on a 16x2 LCD screen. The objective of the design is to help produce a low cost, energy efficient incubator for hatching Japanese quail eggs.
\end{abstract}

\section{Keywords}

Temperature, Humidity, Incubation, Arduino microcontroller,

\section{INTRODUCTION}

In 2016, World meat production rose to 320.7 million tonnes of which poultry accounted for 12.7 million tonnes [1]. Global annual meat consumption per capita is expected to reach 35.3 $\mathrm{kg}$ retail weight equivalent (r.w.e) by 2025 and the rise is mainly from poultry. [2]. The poultry industry exists to promote the United Nations' Sustainable Development Goals (SDGs 2016) number 2: "End hunger, achieve food security and improved nutrition, and promote sustainable agriculture" [3]. The objective of improved nutrition is hindered by protein deficiency; - a major burning global problem.

One emerging solution to this challenge is the meat and eggs from Japanese quail farming, which may be an alternative approach to meeting the protein needs of the poor society [4].

Hatcheries are essential in poultry production and the use of artificial egg hatcheries is a key solution to the expected rise in the poultry industry, but the hatcheries in Ghana are more focused on the production of day-old chicks. The production of quails is a new venture in the Ghanaian poultry industry and there is the need to start quail hatchery to serve interested farmers. In this paper, the design and construction of an efficient 14000 capacity quails (4500 chicken) incubator using Arduino microcontroller at $510 \mathrm{~W}$ rated power, is presented. The system presented here was designed and constructed in an effort to produce a low cost, energy efficient domestic incubator for hatching day old chicks from Japanese quail eggs.

\subsection{System Description and Structure}

The incubator system is a thermal and experimental environment designed and constructed to hatch quails efficiently and affordably. It is a forced air type incubator, which makes temperature and humidity inside it to be more consistent and also facilitates the attainment of desired stable temperature and humidity values more quickly when there is a disturbance or variation in ambient conditions. The eggs are housed inside of the incubator which is $120 \mathrm{~cm} \mathrm{x} 100 \mathrm{~cm} \mathrm{x}$ $140 \mathrm{~cm}$, in volume, on trays $(89 \mathrm{~cm} \times 41 \mathrm{~cm} \times 5 \mathrm{~cm})$ the base of which are made of wire mesh. At the top are four incandescent tungsten bulbs serving as heaters with blower fans mounted on them to aid circulation of the heat produced by the bulbs and to regulate the speed of air in the incubator.

There are ventilation holes on the top and on the sides of the incubator walls which allow the exchange of air between the inside and outside of the incubator. The heating devices (100watts incandescent bulbs) produce heat to raise the incubator temperature to a reference temperature inputted at the set point of the Arduino controller based on the kind of bird being hatched in the incubator. At the same time the fans circulate the hot air throughout the incubator. The structure of the incubator is shown in Figure. 1. showing some devices that are used in the construction of the incubator.

\section{CONDITIONS FOR HATCHING EGGS}

Incubation conditions are the most important factors which affect the hatchability of birds. Temperature, humidity, ventilation and turning during the incubation period significantly affect the hatchability of fertile eggs and chicks' quality [5]. Considerable attention was paid to these parameters in the design of the incubator.

The most important factor among the above mentioned that can have dramatic effect on the hatchability of fertile eggs is temperature.

A developing embryo experiences a temperature that depends on the incubator temperature, embryo heat production, and thermal conductivity of the egg [6]. The accepted temperature ranges for hatching eggs depends on the kind of birds to be hatched. For most poultry species, the optimum incubation temperature ranges between 37.0 and $38.0^{\circ} \mathrm{C}$ and a small deviation from this optimum can have a major impact on hatching success and embryo development [6], [7].

With the egg at the correct temperature, the biological process of incubation commences and the embryo begins to grow, so correct temperature range must be sustained throughout the incubation period [8] in order to achieve a higher rate of hatchability. Studies over the period has shown that an increase or decrease of the temperatures outside the critical range increases the mortality rate of the embryos [7]. Also, incubation temperature has been found to affect the hatchling's thermoregulatory ability, hormone levels, and post hatching growth rate [6]. It is therefore important to model the system temperature well in order to achieve the correct temperature range in the incubator. Section 2.1 presents the thermal parameters of the incubator. 


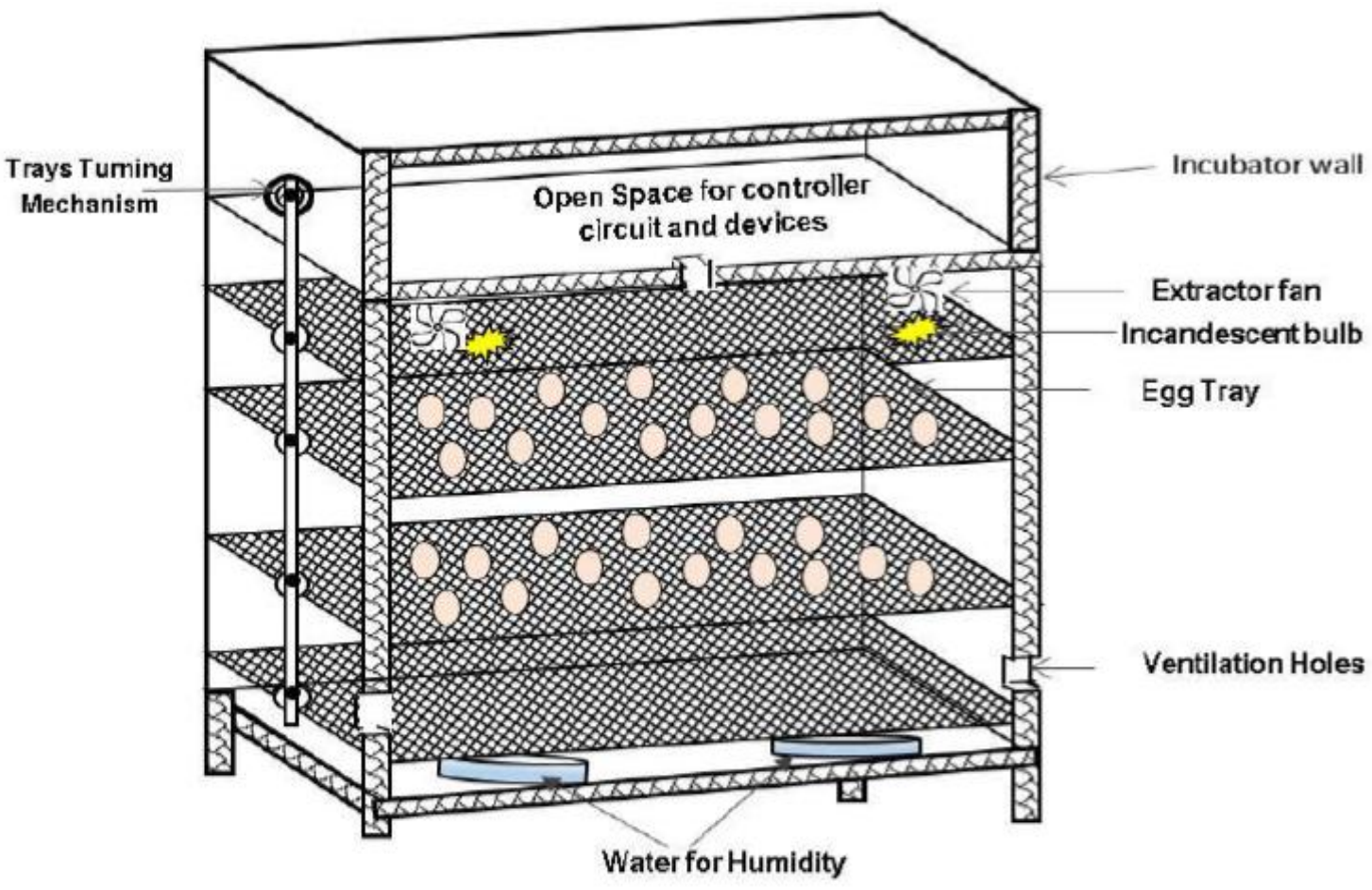

Figure 1. Structure of the incubator showing some devices/equipment in it

Over the incubation period, water passes from the egg through the porous shell of the egg to its environment (that is the internal of the incubator), meaning the internal humidity of the incubator can increase. Correct humidity ensures that the right amount of water is lost over time. It is important to note that there will be variations in the system humidity during the incubation process, but this is not a big problem as far as the variation is within the average humidity range. Decuypere and Michels, in [9] established the relative humidity (RH) of 45$60 \%$ is needed for effective incubation.

The temperature range, the humidity and the air mass flow rate in the incubator are related factors. If the inside temperature of the bird incubator is controlled, the relative humidity is controlled, too [10]. French in [6] presented a simple mathematical model to describe the relationship between the temperature of the developing embryo, incubator temperature, embryo heat production and thermal conductivity of the eggs and its surrounding as in equation (1).

$$
\mathbf{T}_{\mathrm{egg}}=\boldsymbol{T}_{\text {inc }}+\frac{\boldsymbol{H}_{\text {emb }}-\boldsymbol{H}_{\text {water loss }}}{K}
$$

Where

$\mathbf{T}_{\text {egg }}$ is temperature of egg $\left({ }^{\circ} \mathrm{C}\right)$

$\mathbf{T}_{\text {inc }}$ is temperature of incubator

$\mathbf{H}_{\mathrm{emb}}$ is heat production of embryo $(\mathrm{J})$

$\mathbf{H}_{\text {water loss }}$ is heat loss from evaporative cooling (J)

$\mathbf{K}$ is thermal conductance of egg and surrounding boundary $\left(\mathrm{W} /{ }^{\circ} \mathrm{C}\right)$

\subsection{Theory of Heat Exchange in Egg Incubation}

Several researchers have modelled various thermal energy exchanges of incubation based on the equation (1). The heat balance of an embryo is described as follows [5]:

$$
H_{\text {emb }}=H_{\text {water loss }} \pm H_{\text {rad }} \pm H_{\text {conv }}
$$

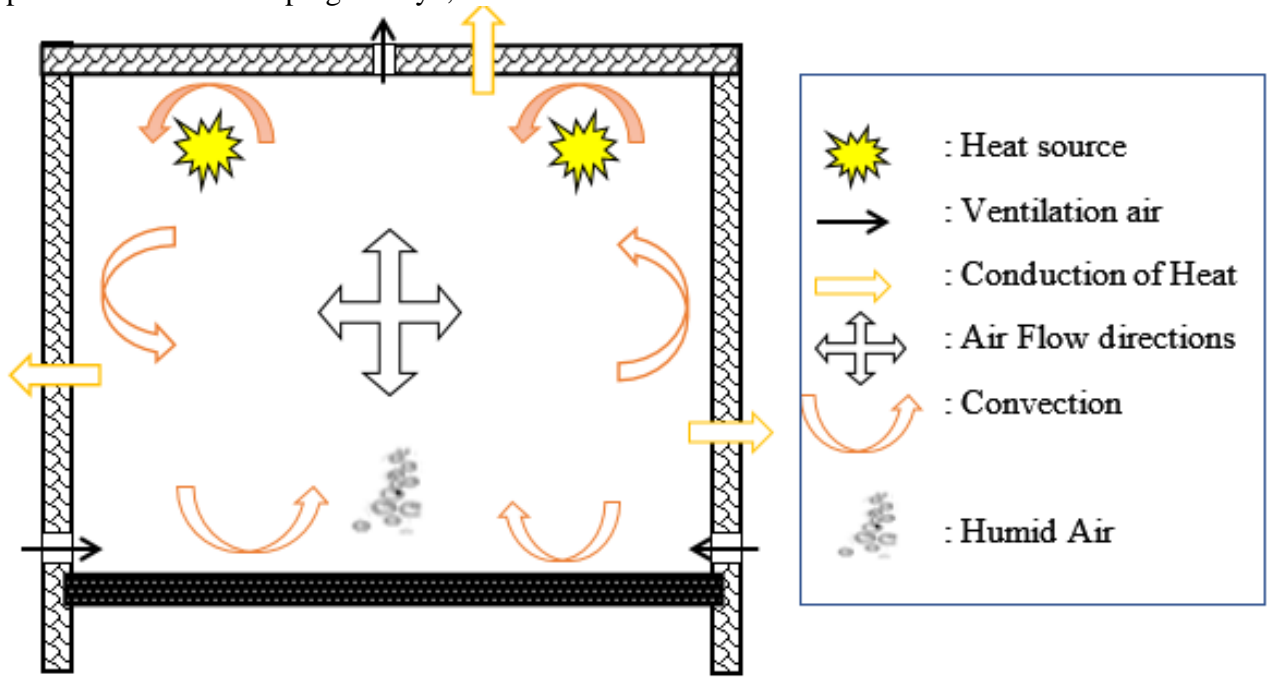

Figure 2. Cross section of the incubator showing the heat and air flow directions [12] 
This can be rewritten as

$$
H_{\text {emb }}-H_{\text {water loss }}=H_{\text {rad }}+H_{\text {conv }}
$$

where $H_{\text {rad }}$ and $H_{\text {conv }}$ are the heat loss or gained by radiation and convection (Joules) respectively.

Equation (1) uses the terms Hemb - Hwater loss to describe the heat loss or gain of an egg because they are easier to measure than either $\mathrm{H}_{\text {rad }}$ or $\mathrm{H}_{\text {conv }}$. Heat transfer through radiation is assumed to be negligible because of the uniform circulation of heat within the incubator and as such all the surfaces within the incubator will be at temperatures close to (within approximately 1 to $2^{\circ} \mathrm{C}$ of) the surface temperature of the egg [6]. In a commercial incubator, an egg would be surrounded by other eggs at the same temperature, thereby reducing the effective radiative surface of the egg [6], [7]. It is therefore assumed that the main transfer of heat between the egg and its surrounding occurs only through convection.

The $\mathrm{H}_{\text {water loss }}$ term caters for the water loss of the embryo. Eggs continually lose water throughout the incubation period, which has been estimated to $12 \%$ of the fresh egg weight between the onset of incubation and the start of pipping [6]. The phase change from liquid water to water vapour requires heat and at incubation temperature this equates to approximately $580 \mathrm{cal} / \mathrm{g}$ of water lost [6], [11].

\section{MATERIALS AND METHODS}

The incubator is a thermal system that is expected to maintain an amount of heat required to achieve high percentage hatchability of any kind of birds incubated in it with high energy efficiency and at an affordable cost. To achieve this, the materials and methods for the construction of the incubator were selected to achieve the conditions as enumerated in section 2 .

\subsection{Materials}

Table 1: List of materials for the construction of the incubator

\begin{tabular}{|l|l|l|}
\hline S/No & Material & Specification \\
\hline 1 & Wire mesh & $\begin{array}{l}1.5 \times 1.5 \mathrm{~cm} \text { Garden fence } \\
\text { ype }\end{array}$ \\
\hline 2 & $\begin{array}{l}\text { Arduino } \\
\text { microcontroller }\end{array}$ & Arduino Uno - R3 \\
\hline 3. & Heat Sensor & DHT22 \\
\hline 4. & Incandescent Bulbs & 100 -watt \\
\hline 5. & Lamp Holder & Screw Type \\
\hline 6. & Relays & $6 \mathrm{~V}, 10 \mathrm{~A}$ \\
\hline 7. & Wiper Motor & \\
\hline 8. & Extractor fans & 30 -watts, 240V \\
\hline 9. & Cables for connection & $1.5 m^{2}$ \\
\hline 10. & Flat iron bar & 1 inch (Thickness) \\
\hline 11. & Angled iron & 1 inch (Thickness) \\
\hline 12. & Square metal pipe & 1 X 1 inch \\
\hline & & \\
\hline
\end{tabular}

The system was constructed from local materials and simple electronic components to make it less expensive. The main structure was built with plywood lined with Formica in order to improve the thermal resistance of the boundary walls. Other materials and items used in the construction is presented in table 1

\subsection{Incubator Structure and Air Flow Phenomena}

The incubator system includes automatic temperature controller, humidifier, and turner subsystems, which set the necessary conditions required for its effective operation. The temperature controller and the humidifier subsystems were modelled to ensure accurate determination of the correct temperature and humidity in the incubator as described in section 2. Another important operation of the system is the turning mechanism which turns the eggs every three hours which is described in section 3.4.

The incubator is a thermal system, because it involves the flow and storage of heat. That is, it is required to maintain an amount of heat for the period of its operation. The heat flow involved here occur through two mechanisms of convection and conduction. The convection mechanism ensures the evenly distribution of heat throughout the incubator as shown in Figure 2. This is achieved by the use of extractor fans which extracts the heat from the sources and circulate it in the incubator.

The conduction occurs through the walls of the incubator due to the differences in the temperature inside the incubator and that of its environment. This leads to heat losses to the environment and has been modelled by equation (5) under subsection 3

\subsection{Modelling the temperature and the control of the system}

Since the incubator in this project is mainly being used to for quail hatching, the value used for modelling the temperature is $37.8^{\circ}$ C. This notwithstanding, the system can be set to different heat levels in order to make it possible for hatching other of bird species.

To achieve this temperature in the $120 \times 100 \times 140 \mathrm{~cm}$ incubator, with tungsten incandescent bulbs, as the heat source, we modelled the temperature control of the system in order to determine the thermal dynamics of the system. This was done with respect to the desired thermal characteristics of the incubator, the ambient environment of the incubator as well as the incubator heating system.

Figure. 3 is a block diagram of the heat flow and the feedback process for the proper control of the incubator. The desired temperature for the incubator is set at the $\mathrm{T}_{\text {set }}$. The heat source controller consists of the Arduino microcontroller and the relays for switching the lights and the blower fans. The disturbance is the heat lost to the ambient environment by conduction through the walls and the ventilation holes. The sensor is DHT22 programming sensor which detects the internal temperature of the incubator and sends a feedback to summing point which compares it with the set point temperature. If the feedback value is lower than the set point value (i.e. $\mathrm{T}_{\mathrm{inc}}<\mathrm{T}_{\mathrm{set}}$ ), an error signal is sent to the heat source controller to close the circuit for more heat to be injected into the incubator. On the other hand, if $\mathrm{T}_{\mathrm{inc}}>\mathrm{T}_{\mathrm{set}}$ the controller switches the heat source off.

The change in the incubator temperature is calculated as

$$
\frac{d T_{\text {inc }}}{d t}=\frac{1}{\left(M_{\text {air }} \times C\right)} \times\left(\frac{d Q_{\text {heater }}}{d t}-\frac{d Q_{\text {losses }}}{d t}\right)
$$

Where:

$$
\begin{aligned}
& M_{\text {air }}=\text { mass of air inside the incubator } \\
& \mathrm{C} \quad=\text { heat capacitance of the incubator } \\
& d Q_{\text {heater }}=\text { heat supplied by the heater. }
\end{aligned}
$$


The environment is modelled as a heat sink with infinity heat capacity and time varying temperature $T_{\text {out }}$. Since the incubator is kept in a room, the average ambient temperature is considered to be $25^{\circ} \mathrm{C}$. The ambient temperature enables us to determine the heat losses to environment. These heat losses and the temperature time derivative are expressed in equation (5)

$$
\left(\frac{d Q}{d t}\right)_{\text {losses }}=\frac{T_{\text {inc }}-T_{\text {out }}}{R_{e q}}
$$

Where:

$$
\begin{aligned}
& \left(\frac{d Q}{d t}\right)_{\text {losses }}=\text { is the heat losses to the environment } \\
& T_{\text {inc }}=\text { the amount of heat required in the incubator } \\
& T_{\text {out }}=\text { the time varying ambient temperature } \\
& R_{\text {eq }}=\text { equivalent thermal resistance of the incubator }
\end{aligned}
$$

\subsubsection{Thermal capacitance of the incubator}

The thermal capacitance of the incubator is the algebraic relationship that exists between the temperature of the physical body and the heat stored within it [13]
If the change in temperature $[\theta(t)]$ in the incubator from initial time $\left(\mathrm{t}_{0}\right)$ to a time $(\mathrm{t})$ is modelled as

$$
\theta(t)=\int_{t_{0}}^{t}\left[Q_{\text {in }}-Q_{\text {out }}\right] d t
$$

Where, $Q_{\text {in }}=$ heat input from the heat source and $Q_{\text {out }}=$ heat losses.

Taking the heat supplied between $\mathrm{t}_{0}$ and $\mathrm{t}$ as a product of a constant $\mathrm{C}$ and a change in temperature as in equation (6) then

$$
\theta(t)=\theta\left(t_{0}\right)+\frac{1}{C} \int_{t_{0}}^{t}\left[Q_{\text {in }}-Q_{\text {out }}\right] d t
$$

Differentiating (7)

$$
\dot{\theta}=\frac{1}{C}\left[Q_{\text {in }}-Q_{\text {out }}\right]
$$

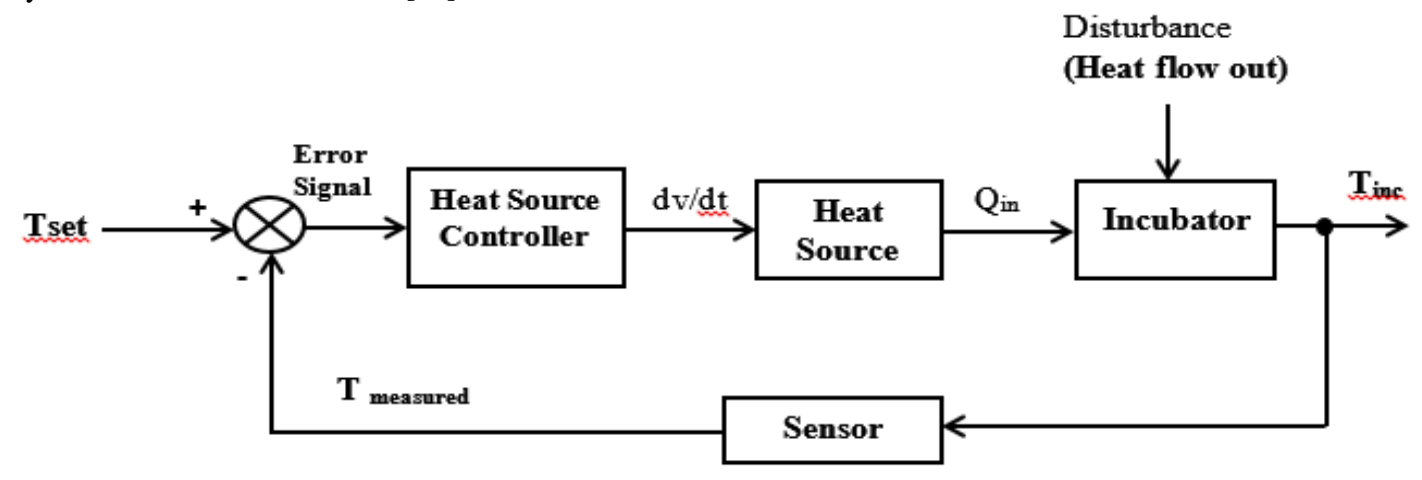

Figure 3. Heat Flow and Feedback Block Diagram

The constant $\mathrm{C}$ is the thermal capacitance of the incubator. We assume the thermal gradient in the incubator is very small as such (8) could be used without dividing the incubator into cells and $\mathrm{C}$ is considered as a whole.

\subsubsection{Heater parameters}

In this work, we explored the inefficiency of the incandescent lamp to our advantage. It is estimated that a 100 -watt bulb is about $10 \%$ efficient for light production [14]. In other words, it produces light with 10 watts of power and 90 watts is converted to heat. So, the main source of heat are four 100watt tungsten incandescent bulbs.

\subsubsection{Heat, Temperature and Humidity control}

The $4-100 \mathrm{w}$ incandescent lamps are well arranged to maintain the required temperature in the incubator within the set range. Extractor fans placed on the lamps pick heat from the bulbs and uniformly distribute it by directing the heat to the walls so that the heat is evenly distributed from the wall. The inside temperature is monitored continuously by the temperature sensor (DHT22). This sensor sends feedback signal to the Arduino microcontroller as shown in Figure 3. The Arduino actuates the relays for the heaters based on the information obtained from the sensor to control the $\mathrm{ON}$ and OFF states of the heat sources (Lamps). If the sensed temperature is less than $37.5^{\circ} \mathrm{C}$ the controller issues $\mathrm{ON}$ signal to bring in more heat. But if the sensed temperature is greater or equal to $37.8^{\circ} \mathrm{C}$ then the controller issues $\mathrm{OFF}$ signal to switch off the heat sources.

\subsection{Egg Tray Turning Mechanism}

Research have shown that the position of the eggs and their rotation influence the loss of water during the incubation, as well as the eclsion percentage [15]. For the rotation, the result of the presented study shows that it is more favourable to rotate the eggs at an angle of $180^{\circ}$. This rotation is necessary to avoid the attachment of the embryo to the egg shell and also to reduce the death rate during the incubation [15].

In this project, the sixteen trays are spaced $3 \mathrm{~cm}$ apart and each is pivoted to a rectangular frame (like louver of windows) which is bolted to the incubator cabinet. A connecting bar couples the tray stands and a DC motor through a gear. As the motor turns the gear, then, the tray stands move up or down like a see-saw. The turning is specifically done at the gear side. The gear is circular, so turning $180^{\circ}$ means up and another $180^{\circ}$ means down. The DC motor is actuated by signal from the Arduino Microcontroller every three hours to automatically rotates the trays through an angle of 15 degrees which cause the eggs to turn 180 degrees.

\subsection{Programming Arduino microcontroller}

Selection of sensor DHT 22 and controller (Arduino) was one of the key to this incubator, even though many of such are available, the Arduino is easy to program and functionalities that makes it easy for automated control. Programming Arduino microcontroller as an incubator controller employs special header files in its coding, such as DHT22 header and LCD header. 
The Arduino microcontroller programming requires knowledge in Arduino programming language (based on wiring), and the Arduino Software (IDE) [16], programming which is similar to $\mathrm{C}$ language, has its own compiler, easy for beginners use, yet flexible enough for advanced users. The programming process involved the programming the LCD, the DHT22 sensor and the control logic on the microcontroller. The flow chart for the program is depicted in Figure 4.

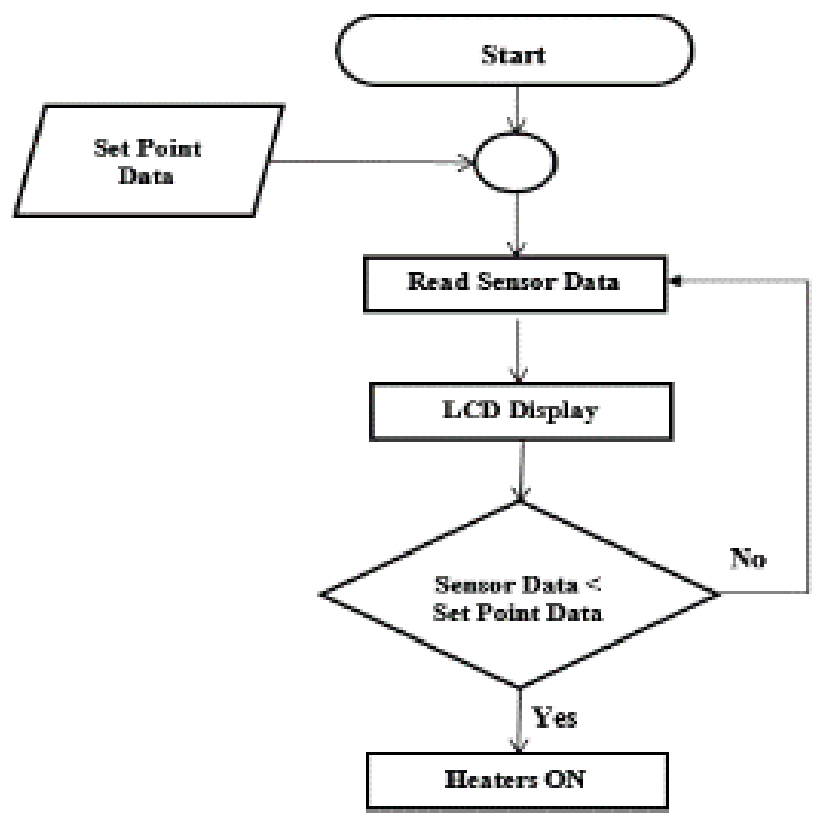

Figure 4. The flow chart for the program

\subsection{System Circuit and connections}

The relay circuit consists of four relays each rated $6 \mathrm{~V}, 10 \mathrm{~A}$; four $100 \Omega$ resistors; four $802 \Omega$ resistors; and four IN4001 diodes. All of them were wired on a PC board. The relays are triggered using a $2 \mathrm{~N} 2222 \mathrm{n}-\mathrm{p}-\mathrm{n}$ transistor actuated by the control signal from the Arduino microcontroller. Two relays are used for switching two of incandescent lamps to a $240 \mathrm{~V}$ supply, while the other two relays are for controlling the blower fans and switching the gear motor for operating the tray turning mechanism respectively. The connection between the various elements making the circuit is shown in Figure 5. The diode rectifier circuit which takes its source from the secondary of the stepdown transformer, serves as a DC supply for the microcontroller. The circuit was simulated and tested with a software called 'Circuit Maker' as shown in Figure 6, before real time implementation.

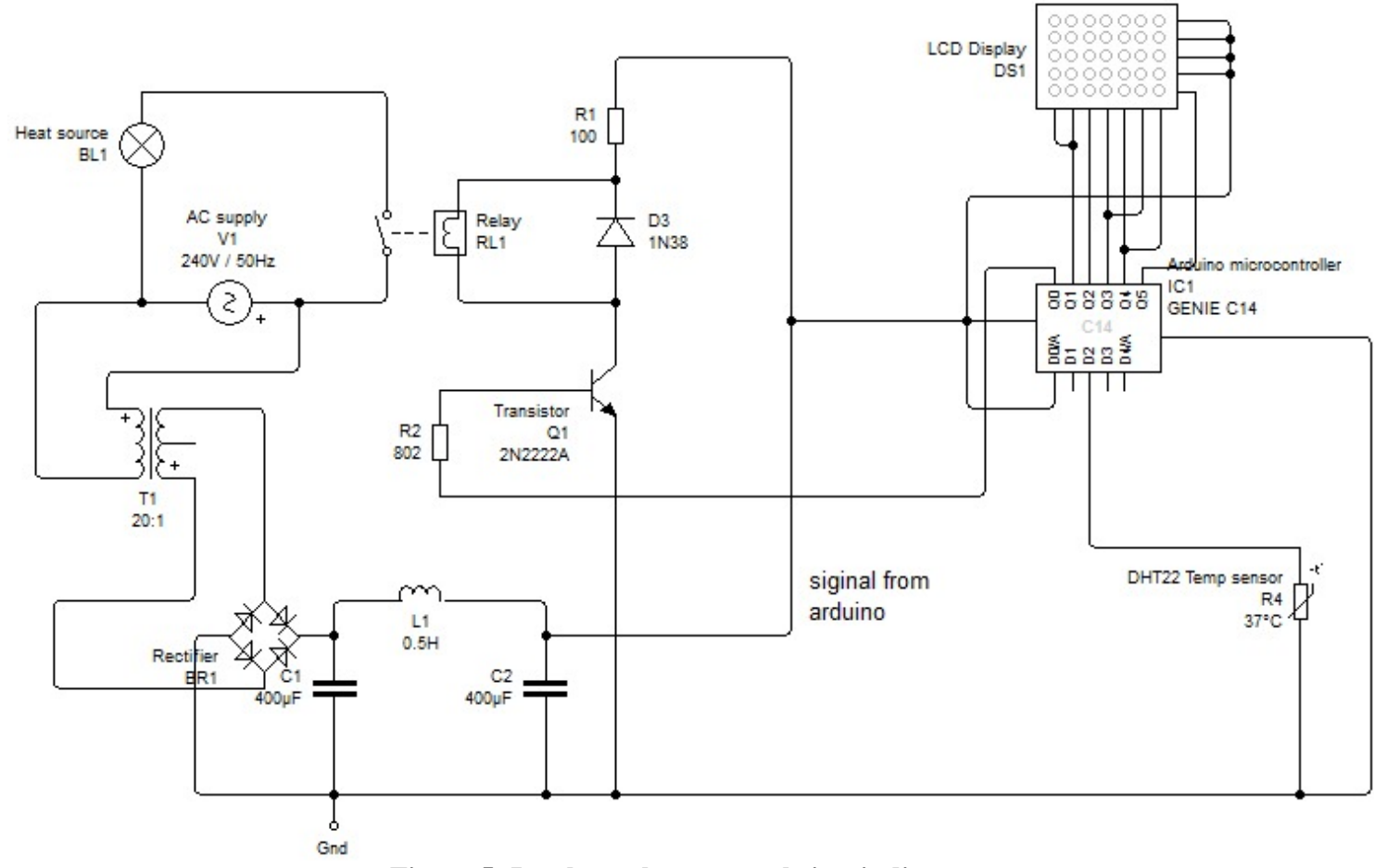

Figure 5: Incubator heat control circuit diagram 


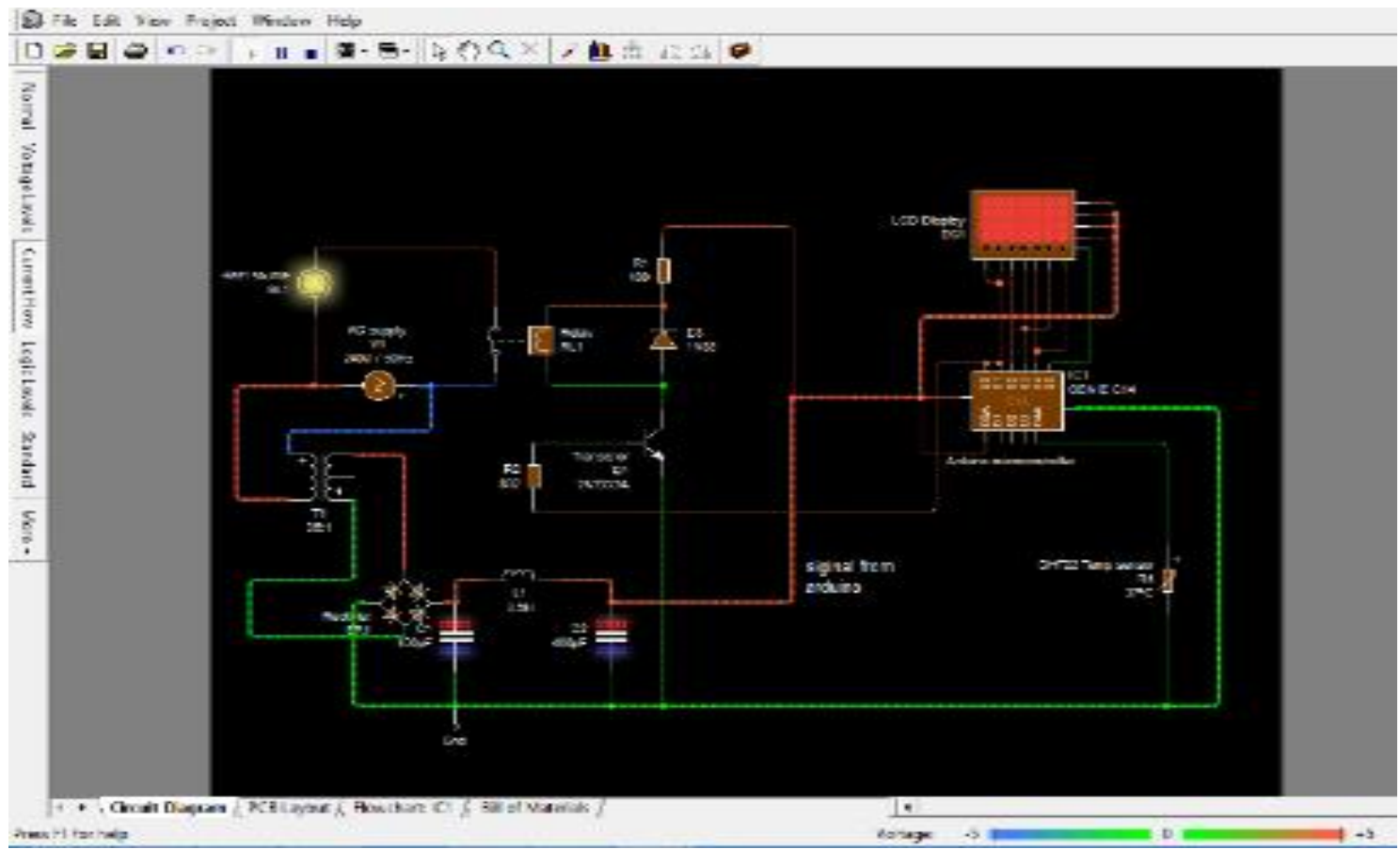

Figure 6: Circuit Simulation

\section{RESULTS AND DISCUSSION}

Figureure 7, shows the designed incubator with the temperature and humidity display and other parts. It has been used to successfully hatch 3000 Quail chicks at 94\% hatchability rate, which is an improved efficiency over those reported in [5] and [17] which achieved hatchability rates of $82.6 \%$ and $33 \%$ respectively.

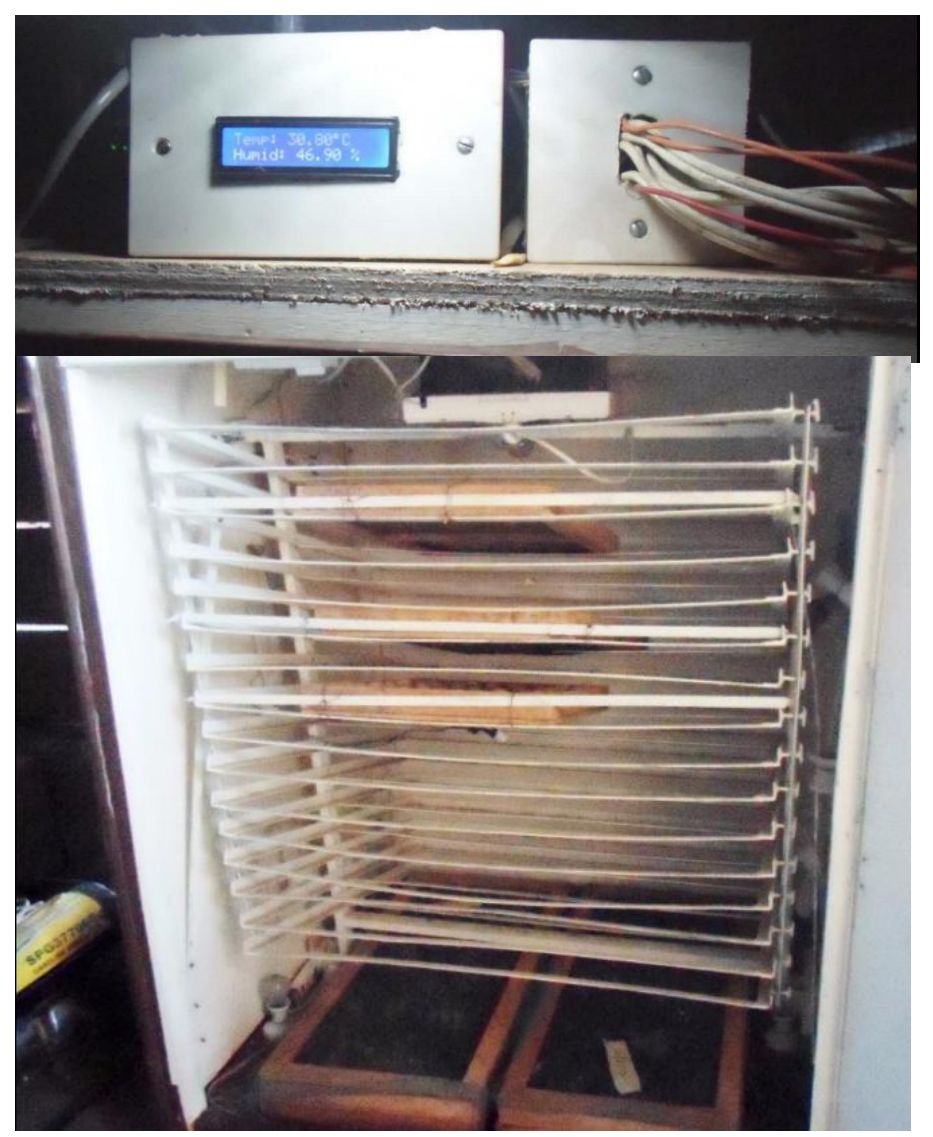

Figure. 7: A Picture of the incubator: Top part- showing the temperature and humidity readings. Below - the tray racks, incandescent lamp and other components inside the incubator 
During the testing period, the system was monitored for a 24hour period, recoding the temperature and the humidity levels in the incubator as displayed by the LCD screen, every two hours. This was to enable the determination of the rate of heat rise from the initial incubator temperature to the steady state temperature of $37.5^{\circ} \mathrm{C}$. It was also to determine the correlation between ambient temperature and humidity levels and the that of the incubator.

Finally, the monitoring enabled us to determine the duty cycle of the heaters in order to ascertain the power consumption of the incubator. Figure 8 shows the correlation between the ambient temperature and

that of incubator, while Figureure 9 is a plot of relation between ambient humidity and that of the incubator.

Firstly, it could be observed from the temperature graph that the temperature in the incubator increased from the initial of $30.5^{\circ} \mathrm{C}$ to $37.1^{\circ} \mathrm{C}$ in the first 2-hours. After this there was not much variation in the incubating temperature from the set point value $\left(37.5^{\circ} \mathrm{C}\right)$. Even though the ambient temperature over the 24-hour period varied between a minimum of $24^{\circ} \mathrm{C}$ and a maximum of $34^{\circ} \mathrm{C}$, the incubator inner temperature remained relatively constant, after the first 2-hours varying between $37.1^{\circ} \mathrm{C}$ and $38.1^{\circ} \mathrm{C}$. This is an indication that heat lost by conduction through the walls of the incubator is minimal.

Secondly, the ambient humidity over the testing period was between $40 \%$ and $73 \%$ while the internal humidity of the incubator varied between $53 \%$ and $70 \%$, also an indication that the designed incubator is able to maintain the averaged relative humidity required for successful hatching.

Also, for observed period, the duty cycle of the heater was $0.25(25 \%)$. That is the heaters were in the ON state for only $25 \%$ of the observation period, which goes to confirm that the incubator has a good thermal capacity and also energy efficient.

Effective turning mechanism made from metal (flat iron, angle iron and square pipe) and a gear coupled to a wiper motor and tray stands helps turning the eggs every three hours. This prevents the embryo from sticking to the shell and this avoids chick mortality.

\section{CONCLUSION}

A 14000 quail eggs (4500 chicken egg equivalent) capacity incubator based on Arduino microcontroller is designed and constructed in this paper. The precise values of temperature and humidity in the incubator at any point in time are displayed on the 16 X 2 LCD screen. With the working of the four relays and control of the incandescent lamp, fans and turning mechanism an energy efficient incubator has been designed and fully implemented

In the future, we envisage the addition of an internet of Things (IoT) system to enable the monitoring and control of the whole system on a $4 \mathrm{G}$ cell phone. This would enable the operator remotely access conditions in the incubator as well as to shut it down or switch it on through a cell phone.

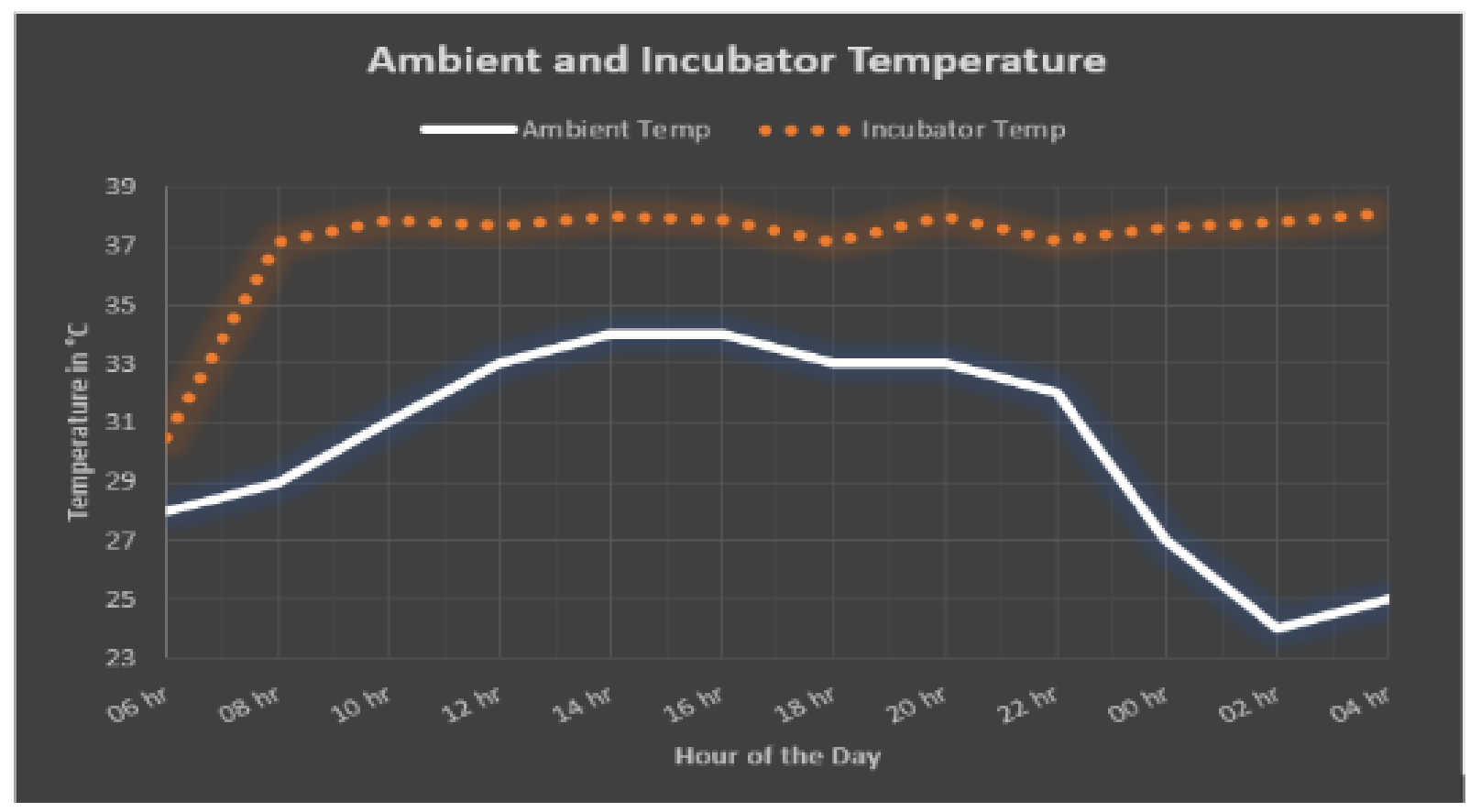

Figure 8: Relationship between Ambient Temperature and Incubator Temperature 


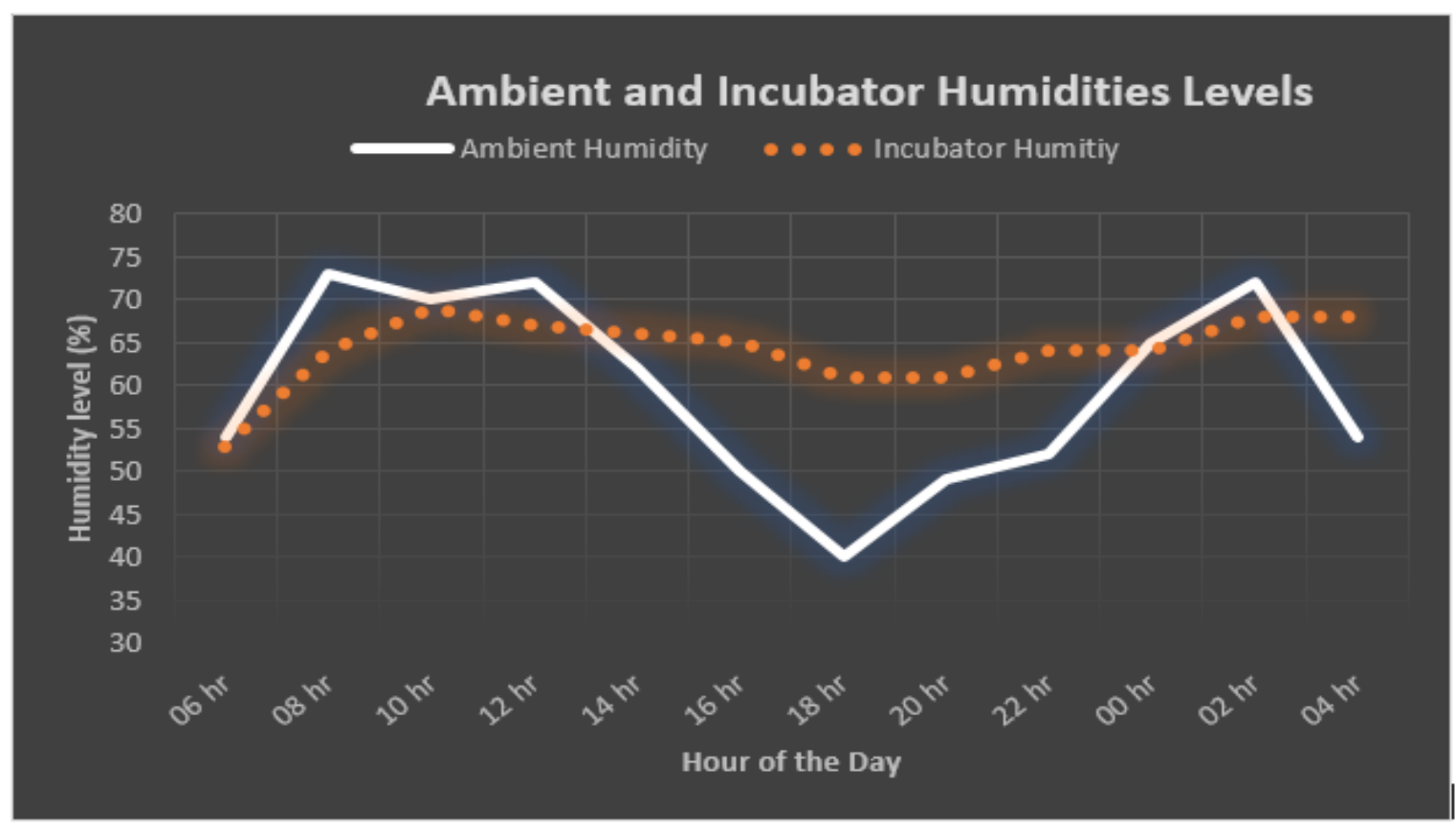

Figure 9: Relationship between Ambient Humidity and Incubator Humidity

\section{REFERENCES}

[1] OECD/FAO, "OECD-FAO Agricultural Outlook 20162025 (Spec ial foc us: Sub-Saharan Africa)," OECD Publishing,, Paris, 2016.

[2] United Nations, "OECD-FAO Agricultural Outlook 2015,” OECD Publishing, Paris, 2015.

[3] United Nations, "The Sustainable Development Goals Report," United Nations, New York, 2016.

[4] Pankaj Deka, Rupam Borgohain, Luit Moni Barkalita, "Design and Evaluation of a Low Cost Domestic Incubator for Hatching Japanese Quail Eggs," International Journal of Livestock Research (ISSN 2277. 1964 ONLINE), vol. 6, no. 1, pp. 92 - 97, 2016.

[5] Harb, S. K., Y. A. Habbib, A. M. Kassem, And A. El Raies, "Energy Consumption For Poultry Egg Incubator To Suituit Small Farmer," Egypt Journal for Agricultural Research, vol. 88 , no. 1, pp. 193 -210, 2010.

[6] N. A. French, "Modeling Incubation Temperature: The Effects of Incubator Design Embryonic Development, and Egg Size," Poultry Science , vol. 76, pp. 124 - 133, 1997.

[7] A. Yılmaz, C. Tepeli , M. Garip , and T. Çağlayan, "The effects of incubation temperature on the sex of Japanese quail chicks," Poultry Science, vol. 90, no. DOI: 10.3382/ps.2011-01471, pp. 2402 - 2406, 2011.

[8] "Incubation guide," The Incubator Shop, 2016. [Online]. Available: http://www.theincubatorshop.co.uk/. [Accessed 8 February 2017].
[9] Decuypere, E. \& Michels, H., “ Incubation temperature as a management tool: a review.," World's Poultry Science Journal , vol. 48, p. 28ะ38. , 1992.

[10] Jose de Jesus Rubio, Martin Salazar, Raul Lugo, Jaime Pacheco, "Modeling of the Relative Humidity and Control of the Temperature for a Bird Incubator," Advances in Computational Intelligence, vol. AISC 61, p. 369-377, 2009.

[11] K. Schmidt-Nielsen, Animal Physiology., New York, NY: Cambridge University Press., 1975..

[12] L. G. Rakotoarimanana, Z. A. Randriamanantany, F. Garde, T. A. Mara, "Building simulation model of an artificial egg incubator during preheat time," 2007.

[13] Charles M Close, Dean K Fredick, Jonathan C. Newel, Modeling and Analysis of Dynamic Systems, New York: John Wiley and Sons, Inc, 2002.

[14] Ezemonye L.I.N, Edeko F.O, Itabor N.A, Olatuji J, Ogbomida E.T, Emeribe C.N, "Energy Efficient Lighting: Luminance Assessments of CFLs and Incandescent Bulbs," Journal of Energy Technologies and Policy, vol. Vol.4, no. No.9, pp. 40 - 46, 2014.

[15] Lucian IONIłĂ, Elena Popescu-Miclosanu, IOAN CUSTURĂ, "A Review of Incubation Parameters in the Japanese Quail (Coturnix coturnix japonica)," Bulletin UASVM Animal Science and Biotechnologies, vol. 67, no. 1-2, pp. 217- 224, 2010. 
[16] "Getting Started | FOUNDATION > Introduction," Arduino AG, January 2017. [Online]. Available: https://www.arduino.cc/en/Guide/Introduction. [Accessed 15th January 2017].

[17] Gbabo Agidi, J.T Liberty, O.N Gunre, G.J Owa, "Design, Construction And Performance Evaluation Of An Electric Powered Egg Incubator," IJRET: International Journal of Research in Engineering and Technology, vol. 03, no. 03, pp $521-526,2014$.
[18] Gregory S. Archer and A. Lee Cartwright, "Incubating and Hatching Eggs," Texas A\&M Agrlife Extension Service, vol. EPS 001, no. 7/13, pp. 1-13.

[19] MathWorks, "Thermal model of a house," Mathworks, Inc. http://www.mathworks.com/help/simulink/examples/thermalmodel-of-a-house.html, 2014. 